\title{
Erratum to: Crowd behavior representation: an attribute-based approach
}

\author{
Hamidreza Rabiee ${ }^{1}$, Javad Haddadnia ${ }^{2 *}$, Hossein Mousavi ${ }^{3}$, Moin $\mathrm{Nabi}^{4}$, Vittorio Murino ${ }^{3}$ and Nicu Sebe ${ }^{4}$
}

\section{Erratum to: SpringerPlus (2016) 5:1179 DOI 10.1186/s40064-016-2786-0}

After publication of this article (Rabiee et al. 2016), we noticed that some of the authors were inadvertently omitted from the author list. These authors, Moin Nabi, Vittorio Murino and Nicu Sebe, have been included in the corrected author list above. Additionally, the images in Fig. 1 have been removed from this article as we did not obtain the necessary permissions to publish them. This does not affect the scientific validity of the article.

\footnotetext{
Authors' contributions

$H R, J H, H M, M N$ and VM designed and created the crowd dataset and provided the corresponding ground-truth labels for videos and frames; $\mathrm{HR}$, JH, $\mathrm{HM}, \mathrm{MN}, \mathrm{VM}$ and NS participated in the design of the study, analyzed data and wrote the paper; HM and MN designed experiments; HR and HM performed experiments. All authors read and approved the final manuscript.

\section{Author details}

${ }^{1}$ Electrical Engineering Department, Hakim Sabzevari University, Sabzevar, Iran. ${ }^{2}$ Biomedical Engineering Department, Hakim Sabzevari University, Sabzevar, Iran. ${ }^{3}$ Pattern Analysis and Computer Vision Department (PAVIS), Istituto Italiano di Tecnologia, Genoa, Italy. ${ }^{4}$ DISI, University of Trento, Trento, Italy.
}

\begin{abstract}
Acknowledgements
This work was supported by the Image Processing and Computer vision Lab of Hakim Sabzevari University, Iran, and Istituto Italiano di Tecnologia, Genova, Italy, and University of Trento, Trento, Italy.
\end{abstract}

\section{Competing interests}

The authors declare that they have no competing interests.

The online version of the original article can be found under doi:10.1186/s40064-016-2786-0.

Received: 25 November 2016 Accepted: 25 November 2016 Published online: 30 December 2016

\section{Reference}

Rabiee H, Haddadnia J, Mousavi H, Nabi M, Murino V, Sebe N (2016) Crowd behavior representation: an attribute-based approach. Springerplus 5(1):1179

\footnotetext{
*Correspondence: haddadnia@hsu.ac.ir

${ }^{2}$ Biomedical Engineering Department, Hakim Sabzevari University,

Sabzevar, Iran

Full list of author information is available at the end of the article
}

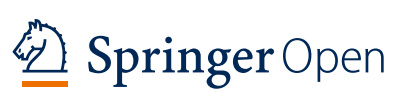

C The Author(s) 2016. This article is distributed under the terms of the Creative Commons Attribution 4.0 International License (http://creativecommons.org/licenses/by/4.0/), which permits unrestricted use, distribution, and reproduction in any medium, provided you give appropriate credit to the original author(s) and the source, provide a link to the Creative Commons license, and indicate if changes were made. 